\title{
Stimulus salience and asymmetric forgetting in the pigeon
}

\author{
JOHN T. WIXTED and SANTINO C. GAITAN \\ University of California, San Diego, La Jolla, California
}

\begin{abstract}
Pigeons were trained using a symbolic delayed matching-to-sample procedure involving bright versus dim houselight samples. We hypothesized that when sample stimuli differ in salience, increasing the size of the retention interval will affect performance on trials initiated by the more salient sample only. In agreement with this prediction, accuracy following the dim sample did not decline as the retention interval increased, whereas accuracy following the bright sample declined to well below $50 \%$ correct. In a second experiment, the less salient (dim) sample from Experiment 1 was arranged as the more salient sample in a sample/no-sample procedure. Accuracy on dim sample trials then declined to well below 50\% correct as the retention interval increased, whereas accuracy on no-sample trials remained constant. The results suggest that when sample stimuli differ in salience, pigeons may transform the nominal discrimination task into a detection task in which they respond on the basis of the presence or absence of the more salient sample.
\end{abstract}

In a typical delayed matching-to-sample (DMTS) procedure involving pigeons, trials are initiated by presenting one of two samples (e.g., red or green), followed by a delay, followed by the simultaneous presentation of two comparison stimuli (e.g., red and green). A response to the matching comparison stimulus is reinforced, while a response to the nonmatching comparison stimulus terminates the trial without reinforcement. In the vast majority of experiments, the two samples involved in the DMTS task are of equal salience.

Several variants of the standard DMTS task involve samples of unequal salience, although differences in salience are not usually considered as an explanation for why the results turn out as they do. Perhaps the most extreme variant of this kind is a presence versus absence procedure in which half the trials are initiated by a salient sample (e.g., a keylight), and the other half involve no sample at all (e.g., Grant, 1991; Wixted, 1993). On these no-sample trials, the intertrial interval (ITI) blends seamlessly into the delay interval. At the end of every trial, two comparison stimuli are presented simultaneously. A response to one comparison is reinforced on sample trials, and a response to the other is reinforced on what can be termed "no-sample" trials.

In less extreme cases, the samples might consist of food versus no-food (where no-food actually consists of the brief illumination of an empty hopper), or a shortduration houselight (e.g., $2 \mathrm{sec}$ ) versus a long-duration houselight (e.g., $10 \mathrm{sec}$ ), or a white keylight that requires

These experiments were conducted while the second author was an NSF Predoctoral Fellow, and the research was supported by NIMH Grant MH55648. Correspondence concerning this article may be directed to J. T. Wixted, Department of Psychology, 0109, University of California, San Diego, La Jolla, CA 92093-0109 (e-mail: jwixted@ucsd.edu).
40 keypecks to extinguish it versus a white keylight that requires only 10 keypecks (Colwill, 1984; Fetterman \& MacEwen, 1989; Sherburne \& Zentall, 1993; Spetch \& Wilkie, 1983). In all of these cases, the forgetting functions following the two samples are asymmetric. Typically, performance following the less salient sample (e.g., no sample, no food, a short sample, or a sample requiring fewer keypecks) begins at a relatively high level and remains accurate as the delay interval increases. Performance following the more salient sample (e.g., in a sample/nosample procedure, presentation of food, a long sample, or a sample requiring more keypecks) decreases rapidly as the retention interval increases and eventually falls to well below $50 \%$ correct. Figure 1 illustrates the typical pattern using data from a sample/no-sample procedure, but a similar pattern has been observed in all of the asymmetric cases mentioned above. By contrast, the use of equally salient samples typically yields symmetric forgetting functions such that performance for both samples declines to chance levels at approximately the same rate.

Why is the asymmetric pattern shown in Figure 1 typically observed when asymmetric samples are employed? The answers offered by previous researchers tend to differ depending on the specific procedure employed. To explain the asymmetric pattern produced by the sample/nosample procedure, for example, Grant (1991) proposed a default response model according to which pigeons choose one comparison stimulus by default but choose the other if memory for the sample happens to be present. Thus, performance on sample trials eventually falls to below $50 \%$ correct because, when the hypothesized memory trace fades completely, pigeons execute their default responses and select the wrong comparison most of the time.

Spetch and Wilkie (1983) explained the same asymmetric pattern produced by an event-duration discrimi- 


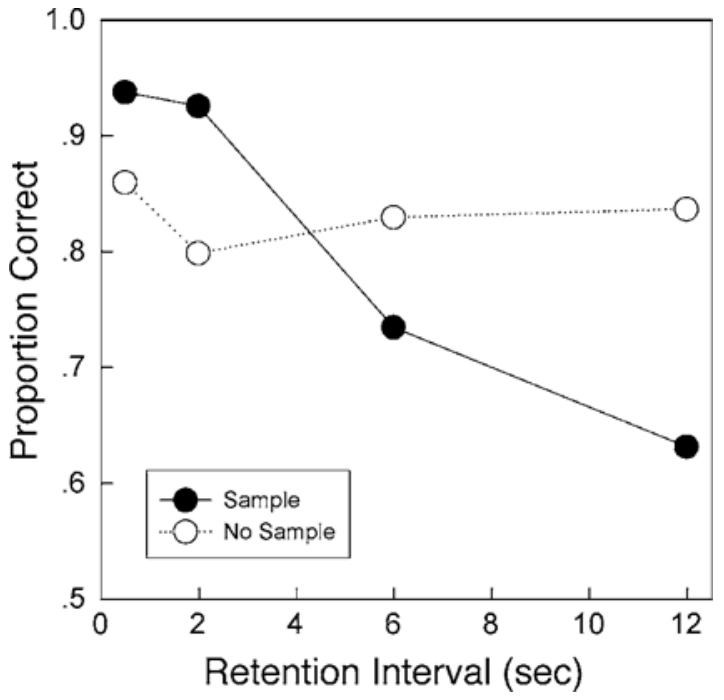

Figure 1. Representative data from Wixted (1993) illustrating the typical pattern of results observed on a delayed presence versus absence discrimination procedure.

nation procedure by proposing that the representation of the duration of a sample shrinks with the passage of time. Thus, immediately after it is presented, a long-duration sample will be accurately represented (leading to accurate performance). As the delay increases, however, that representation will continuously shrink. Eventually, when the delay is long enough, the long duration sample will actually be remembered as having been short in duration. At that point, the pigeon would select the choice alternative associated with the short sample even though the trial actually began with the long sample. The theoretical decrease in the representation of event duration with the passage of time is known as "subjective shortening" (Spetch \& Wilkie, 1982, 1983).

To explain the asymmetric pattern observed when the DMTS "sample" consists of 40 versus 10 pecks to a white key, Fetterman and MacEwen (1989) tentatively proposed an account similar to the subjective shortening account advanced by Spetch and Wilkie (1982). According to this idea, the "choose small" effect results from a reduction in the number of represented keypecks as the delay interval increases.

Although multiple explanations have been advanced in the past, another possibility is that the asymmetric pattern of forgetting arises for the same reason in all these tasks. That explanation was originally advanced to account for performance on a presence versus absence task. Wixted (1993) proposed a signal-detection account of performance on this task that is similar to the default response model in certain respects, but it does not assume that responses are ever made by default. The detection model assumes that, on every (sample or no-sample) trial, pigeons select one comparison stimulus when the subjective "strength of evidence" that a sample occurred exceeds a decision criterion and they select the other when it falls below the criterion. Due to inherent variability, the strength of evidence on a no-sample trial may occasionally exceed the criterion, leading to an incorrect choice. However, because there is nothing to forget on these trials (because nothing was presented), the retention function remains flat. On sample trials, by contrast, memory for the sample is strong following a short delay, which allows the pigeons to make the correct choice on that basis. After a long delay, memory for the sample will have faded in such a way that the strength of evidence that a sample occurred falls below the criterion most of the time. At that point, the sample trial is psychologically equivalent to a no-sample trial, and the pigeons choose the wrong alternative more than $50 \%$ of the time.

This account was designed to explain the asymmetric pattern of forgetting observed on the sample/no-sample task, but it may also explain why that pattern is produced by other tasks as well. Gaitan and Wixted (2000) presented evidence suggesting that birds can transform a nominal short versus long discrimination task into a presence versus absence detection task. In a typical event duration discrimination task, samples consist of either a 2 -sec or a 10 -sec houselight presentation. Given the procedural requirements, it is usually assumed that birds learn to choose one alternative (e.g., red) when they remember a 2-sec sample and choose the other alternative (e.g., green) when they remember a 10 -sec sample. Another possibility is that the birds do not adopt a discrimination strategy like this but instead adopt a detection strategy based on the presence or absence of the more salient sample. According to this idea, pigeons select one alternative when strength of evidence that the more salient sample (e.g., the 10-sec sample) appeared exceeds a criterion and they select the other when it falls below the criterion. If so, then the less salient sample (e.g., the 2-sec sample) is actually superfluous and could be omitted from the procedure altogether without affecting performance. In agreement with this idea, Gaitan and Wixted showed that for birds that exhibit the asymmetric forgetting pattern ( 5 out of 8 birds in their Experiment 1), replacing the 2-sec sample trials with no-sample trials for five sessions and then reinstituting the 2 -sec sample trials for five sessions had no measurable effect on performance.

If the birds transformed the discrimination task into a detection task, why did they respond on the basis of the presence or absence of the 10-sec sample rather than the 2 -sec sample? They did so presumably because the 10 -sec sample is more salient than the 2-sec sample, and the birds may simply find it easier to respond on the basis of its presence or absence. If this account is correct, then the asymmetric pattern observed on the duration discrimination procedure should also be observed on other tasks in which the samples differ in salience. The purpose of the two experiments presented below was to test this prediction by manipulating salience in an obvious way - namely, by using samples that differ in brightness. In a brightness discrimination task, a response to one comparison would be correct on trials initiated by the 
bright sample, while a response to the other comparison would be reinforced on trials initiated by the dim sample. If pigeons are inclined to rely on a detection strategy when the samples are of unequal salience, then performance following the bright sample should decrease rapidly as the post-sample delay increases (and fall to well below $50 \%$ correct), while performance following the dim sample should remain essentially flat. If such a "choose dim" bias develops at increasing delays on the brightness discrimination task, then it would lend further support to the idea that birds can respond on the basis of a detection strategy (whether the more salient sample occurred or not) rather than on the discrimination strategy that the experimenter had in mind (whether the more salient or the less salient sample occurred).

The use of samples differing in brightness is common in DMTS research (e.g., McCarthy \& Davison, 1991). However, in most cases, the data are collapsed across the samples because the effect of brightness is not of central interest. To our knowledge, the research that came closest to testing the question of interest here was performed by Kraemer, Randall, and Brown (1997). They used both long and short samples of both a bright white light and a dim red light. The typical asymmetric pattern of forgetting (i.e., more rapid forgetting for the longer sample) was observed for both bright (white) and dim (red) samples. These results may mean that a long sample is more salient than a dim sample, whether the samples are both bright or both dim. What is not clear from these results is whether a choose dim effect would be observed when samples consist of two equal-duration, same-color lights that differ only in brightness (and no duration discriminations were required).

\section{EXPERIMENT 1}

In our Experiment 1, birds were trained to discriminate a dim houselight from a bright houselight following a short retention interval. Once they had established a criterion level of accuracy, four probe sessions were introduced in which retention intervals of up to $12 \mathrm{sec}$ were presented. Assuming that the bright stimulus is more salient than the dim stimulus, we hypothesized that an asymmetric pattern of forgetting would be observed as the delay from sample presentation increased (the same pattern that has been repeatedly observed in standard presence versus absence and duration-discrimination tasks). More specifically, there should be little or no decline in accuracy following a dim sample as the retention interval increases, whereas accuracy should decline rapidly following the bright sample. In Experiment 2, birds were trained on a dim versus nothing (i.e., presence vs. absence) task. Here, the dim houselight was the more salient sample (relative to nothing), so performance following that sample should have decreased rapidly as a function of the delay interval (instead of remaining flat, as it should have in Experiment 1), whereas performance on no-sample trials should have remained flat.
It is important to emphasize that these experiments were designed to test the hypothesis that samples of differential salience yield predictably asymmetric retention functions. Symmetry (or an asymmetry other than the predicted one) in this new domain would weigh against the claim that differential salience matters much in studies employing differential durations, differential sample requirements, food versus no-food samples, and so forth. The presence of the predicted asymmetries would not only suggest that differential salience may be important in those studies but would also reinforce the theoretically interesting claim that pigeons can transform a discrimination task (e.g., bright vs. dim) into a detection task (e.g., detecting whether or not the bright sample was presented). If pigeons transformed the task in that manner, it would mean that the less salient sample was a superfluous component of the procedure.

\section{Method}

Subjects. Eight White Carneaux pigeons were maintained at approximately $80 \%$ of their free feeding weights. The birds were weighed before each experimental session and $30 \mathrm{~min}$ after the completion of each session were fed an amount of milo and pigeon chow to maintain $80 \%$ weight. Water and grit were available ad lib in the home cages. The colony room was kept on a 16:8-h light:dark cycle. All eight birds were either experimentally naive or had not participated in a research study within the previous two years. One bird became blind during the course of the experiment and was excluded, so only data from the seven birds that completed the experiment are presented.

Apparatus. The experimental chambers used were $35 \mathrm{~cm}$ long, $32 \mathrm{~cm}$ wide, and $34 \mathrm{~cm}$ high. The chambers were constructed of Plexiglas except for one wall that was aluminum. Three Plexiglas response windows, each $6.5 \mathrm{~cm}$ high and $4.4 \mathrm{~cm}$ wide, were mounted in the aluminum wall of each chamber $20 \mathrm{~cm}$ above a wire mesh floor (in both experiments, choice stimuli only appeared on the outside two keys and never on the middle key). An SVGA color monitor, directly visible through the response key windows, displayed the choice stimuli. The distance from the response surface to the monitor surface was approximately $3 \mathrm{~cm}$. The sample stimuli consisted of a 1.2-W, .040-amp overhead houselight located on the ceiling $8 \mathrm{~cm}$ from the rear wall, with a filament luminance of $75 \mathrm{can}-$ dles per square inch for the bright sample, and 4 candles per square inch for the dim sample. Both stimuli had a duration of $5 \mathrm{sec}$. The bright stimulus was powered by a direct connection to a $28-\mathrm{V}$ DC power supply, whereas power to the dim stimulus passed through a $680-\Omega .5-\mathrm{W}$ resistor. In determining appropriate intensities for the samples, human judgment was used, given the relative similarity between human and pigeon perception of light intensity (Schneider, 1987).

Two choice stimuli appeared on each trial, the first consisting of two, vertically stacked, $2.5-\mathrm{cm}$ red circles, and the other consisting of five 1.5-cm green squares arranged in a checkerboard pattern. Both choice stimuli were presented on a $6-\mathrm{cm}$ square white background. Keypecks were recorded via a microswitch operated through depression of the hinged Plexiglas window. A small pellet tray located below the center window and $4 \mathrm{~cm}$ above the chamber floor collected Noyes 45-mg food pellets from a Gerbrands precision pellet dispenser (two pellets per reinforcement). The chambers were not illuminated, apart from sample presentations and pellet tray illumination during reinforcement. Each chamber and respective VGA monitor were enclosed in a sound- and light-attenuating chamber, and exhaust fans masked extraneous noise. An IBM-compatible computer controlled the experiment and recorded the data. A closed- 

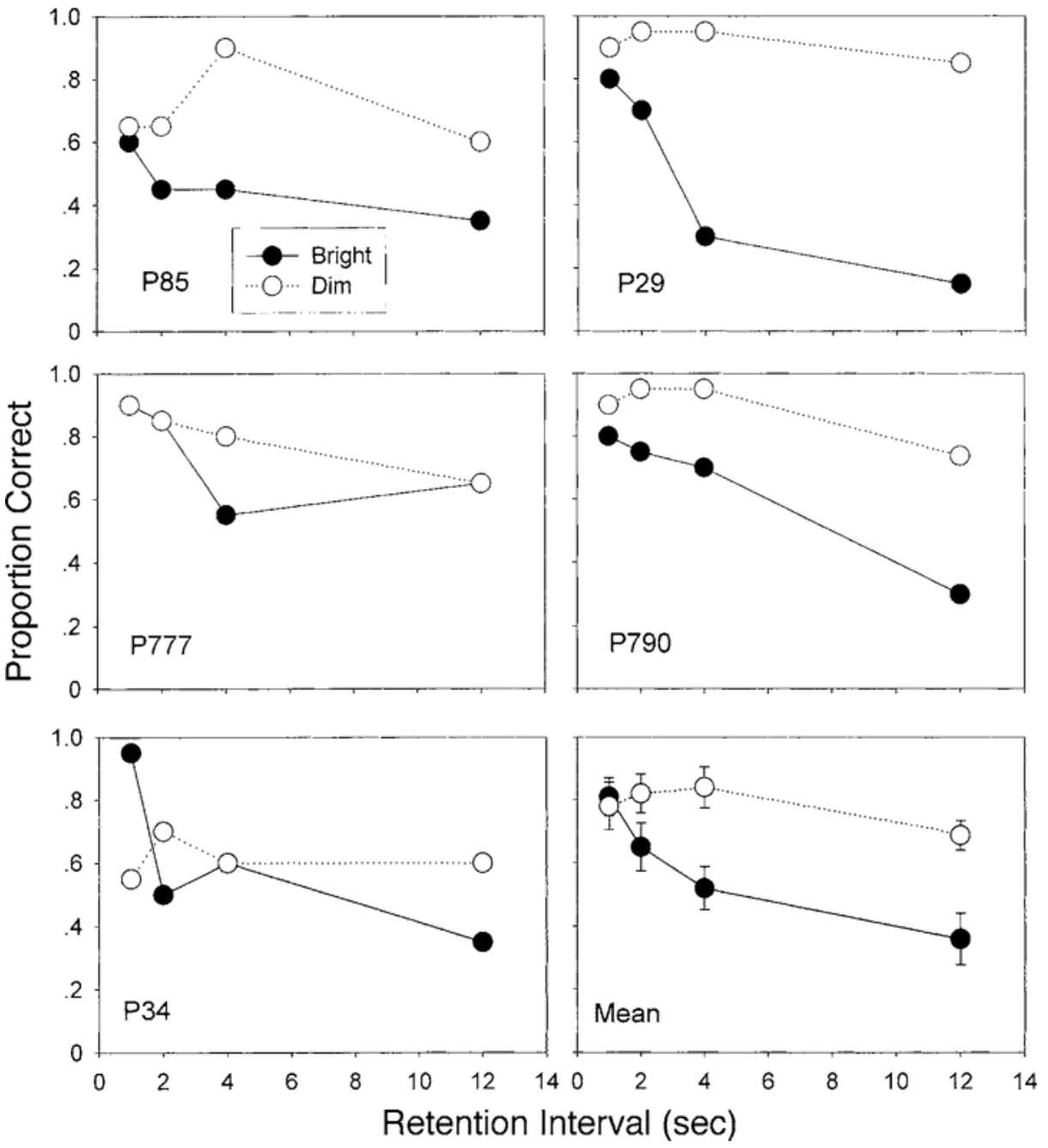

Figure 2. Proportion of correct responses as a function of retention interval on the dim and bright sample trials for the 5 birds in Experiment 1 that reached criterion over four sessions. These probes were conducted at a $75 \%$ criterion level of performance.

circuit video camera and microphone inside each chamber allowed visual and aural monitoring of the birds.

Procedure. Initial training consisted of 40 trials evenly divided between dim and bright houselight trials. The red and green choice stimuli were presented after a 1-sec delay following termination of the sample. The choice stimuli were displayed on the left and right choice keys with positioning randomly determined for each trial. A peck to one key light (green for 4 birds, red for the other 4) was reinforced with two food pellets and a 5-sec hopper light following dim samples. A peck to the other choice key was similarly reinforced following presentation of the bright stimulus. Termination of the hopper light or an incorrect choice initiated an ITI of $30 \mathrm{sec}$ Order of stimulus presentation was determined randomly at the beginning of each session, although during initial training a correction procedure was used such that incorrect responses resulted in repetition of the trial until a correct response was given (e.g., until the pigeon pecked the correct choice stimulus on the opposite key).

When performance was consistently above chance, the correction procedure was halted. Training continued until an average overall accuracy of $75 \%$ correct was maintained across five consecutive sessions, after which probe sessions were introduced. The probe sessions differed from the baseline sessions only in the variability and length of the retention interval. Whereas baseline sessions used a retention interval of $1 \mathrm{sec}$ on every trial, probe sessions involved retention intervals of $1,2,4$, and $12 \mathrm{sec}$. In each probe session, the four retention intervals each occurred four times for both the dim and bright stimuli and were ordered randomly. A total of four sessions including probes were run for each bird, with probe sessions separated by two sessions of baseline training in which all retention intervals were again $1 \mathrm{sec}$ long. Baseline training was then reinstated until an average overall accuracy of $85 \%$ correct was maintained across five consecutive sessions, after which a second set of four retention interval probe sessions was conducted identically to the first series described above.

After completion of the second series of probes, the baseline training of dim and bright samples at 1-sec retention intervals was reinstated for approximately 4 weeks. At this point, a series of nohouselight, nonreinforced sample probes was introduced. These nosample probes were intended to be as unobtrusive as possible. Toward this end, only four no-houselight probes were given, randomly interspersed among the normal 20 dim sample trials and the 20 bright sample trials (for a total of 44 trials). Three probe sessions were conducted for each bird, with two sessions of normal baseline 
training in between each session with probes. Although all of the birds had been exposed to retention intervals as long as $12 \mathrm{sec}$ in the preceding phases, none had been presented explicit no-houselight samples before this time. On no-houselight probe trials, the end of the ITI blended seamlessly into the 1 -sec retention interval.

\section{Results and Discussion}

All 7 birds learned the bright/dim discrimination to above chance levels, but only 5 met our criterion of $75 \%$ correct for five consecutive sessions. These 5 birds were exposed to retention interval testing, and the results for each bird are shown in Figure 2.

Pigeons 29 and 790 show the predicted asymmetry in that performance for the dim sample remains relatively unaffected by the size of the retention interval, whereas performance for the bright sample decreases rapidly. Pigeon 85 shows a smaller asymmetry in the predicted di- rection, whereas the remaining 2 birds do not exhibit an asymmetry. Despite some variability at the individual level, however, performance averaged across all 5 birds (lower right panel of Figure 2) shows a statistically significant interaction between retention interval length and stimulus intensity $[F(3,9)=5.8, p=.011]$.

Figure 3 presents the results for each bird from the probe sessions after baseline performance reached a criterion of $85 \%$ correct. At the group level, the results appear to be nearly identical to the results obtained when the birds were tested after they had reached the criterion of $75 \%$ correct. As before, the predicted interaction between brightness level and retention interval was significant $[F(3,9)=20.1$, $p<.001]$. Although the group mean data remained largely unchanged, a choose-dim effect was evident for all 5 birds.

The results of the no-sample probes are presented in Figure 4. All 5 pigeons showed a strong preference for
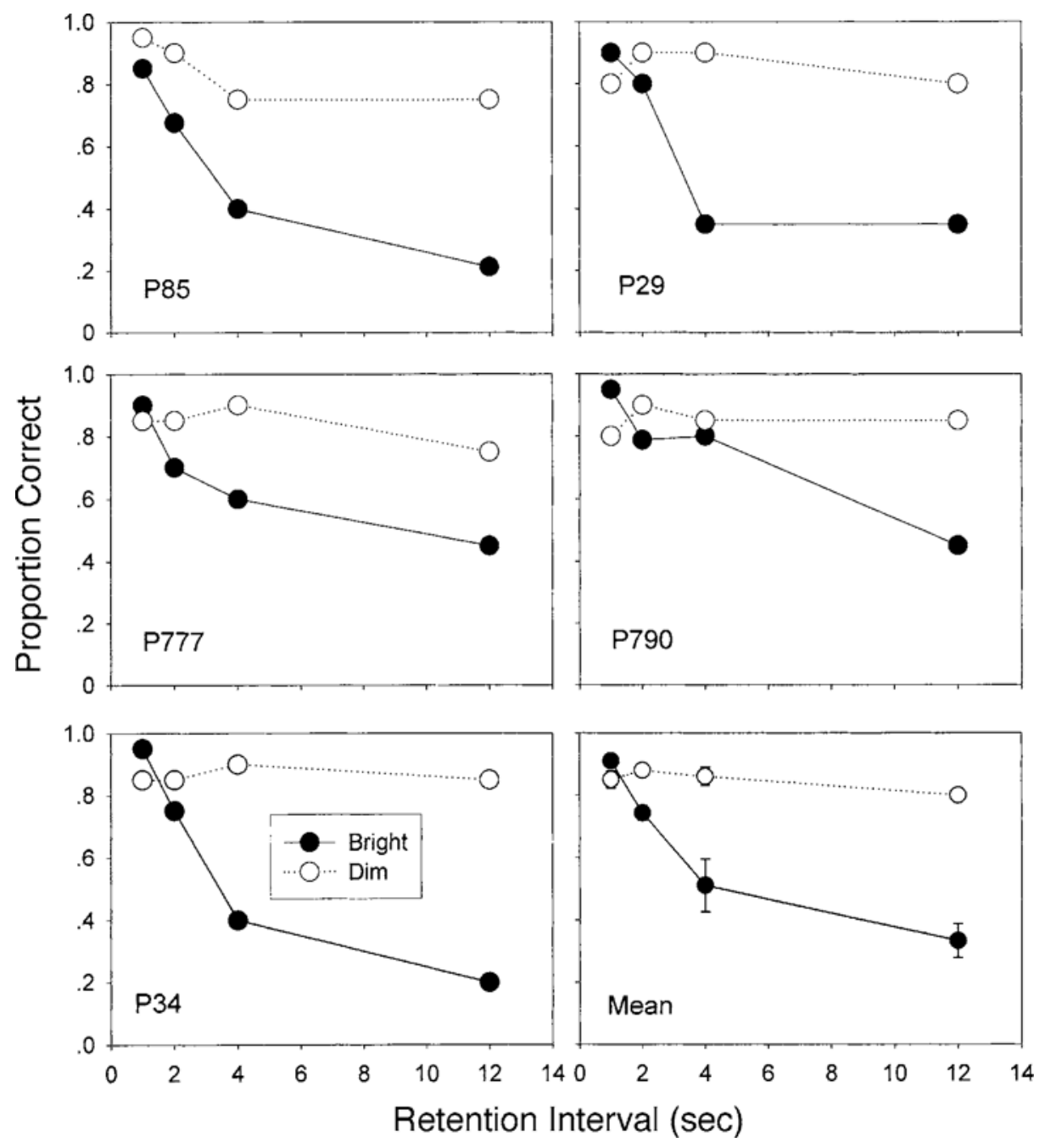

Figure 3. Proportion of correct responses as a function of retention interval on the dim and bright sample trials for the 5 birds in Experiment 1 that reached criterion over four sessions. These probes were conducted at an $85 \%$ criterion level of performance. 


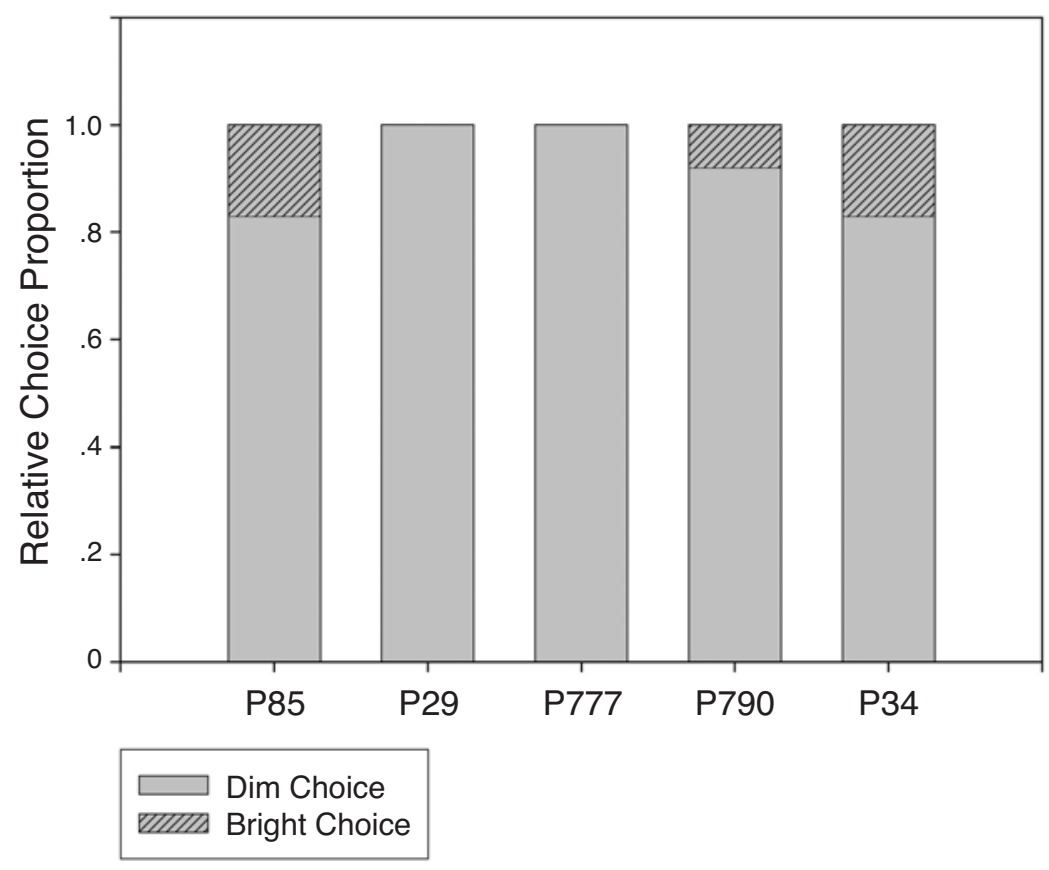

Figure 4. Choice stimulus preference following nonreinforced no-houselight (nosample) probes introduced at the end of Experiment 1.

the choice stimulus associated with the dim stimulus when no sample was presented. Overall, the birds chose the dim-associated comparison stimulus on $91.6 \%$ of the no-sample probe trials, which is about how often they chose that comparison on trials that actually began with the dim stimulus. For example, over the last five sessions prior to retention interval probe testing at the $85 \%$ criterion level, the birds responded correctly on dim sample trials $90.3 \%$ of the time, a value that does not differ significantly from that observed on no-sample trials.

The comparable values obtained on dim trials and on no-sample trials is as it should be if the birds were responding on the basis of the presence or absence of the bright sample. The bright sample is equally absent whether the trial begins with a dim sample or no sample. Note that performance on our no-sample probe trials corresponds to the typical pattern observed in prior studies involving samples that differ in salience (e.g., Fetterman \& MacEwen, 1989; Spetch \& Wilkie, 1983). In each case, birds were shown to choose the alternative associated with the less salient sample when no-sample probes were introduced.

\section{EXPERIMENT 2}

Salience is a relative concept. In Experiment 1, the bright sample was salient relative to the dim sample. In Experiment 2, that same dim sample was arranged to be the salient event by using a dim/no-sample procedure. That is, one choice alternative was correct on trials initiated by the dim sample, and the other choice stimulus was correct on trials that involved the absence of a sam- ple. Here, the very same sample that yielded a flat retention function in Experiment 1 should yield a steeply declining retention function that would fall to below $50 \%$ correct (whereas a flat retention function should be observed on no-sample trials).

This experiment also serves to address one possible concern about Experiment 1. The concern is that the dim sample was just too dim to be detected by the birds. If so, then the bright versus dim discrimination task could not have yielded results different from a presence versus absence task. If the dim sample was simply too dim to be discriminated from nothing, then the birds in Experiment 2 should not be able to perform the dim versus nothing task. If the birds are able to discriminate dim from nothing, then the effect of increasing the retention interval should yield the standard pattern for a presence versus absence procedure. That is, performance following the salient sample (which, in contrast to Experiment 1, has become the dim sample) should drop rapidly to a level below $50 \%$ correct, whereas performance following the nonsalient sample (in this case, nothing) should remain flat at a level well above $50 \%$ correct.

\section{Method}

Subjects. Four naive White Carneaux pigeons were maintained at approximately $80 \%$ of their free feeding weights and were generally treated in the same manner as the birds in Experiment 1.

Apparatus. The experimental apparatus was the same as that used in Experiment 1.

Procedure. The training procedure was identical to that of Experiment 1, except that no-sample trials were used in place of the bright sample. On a no-sample trial, the end of the ITI blended 


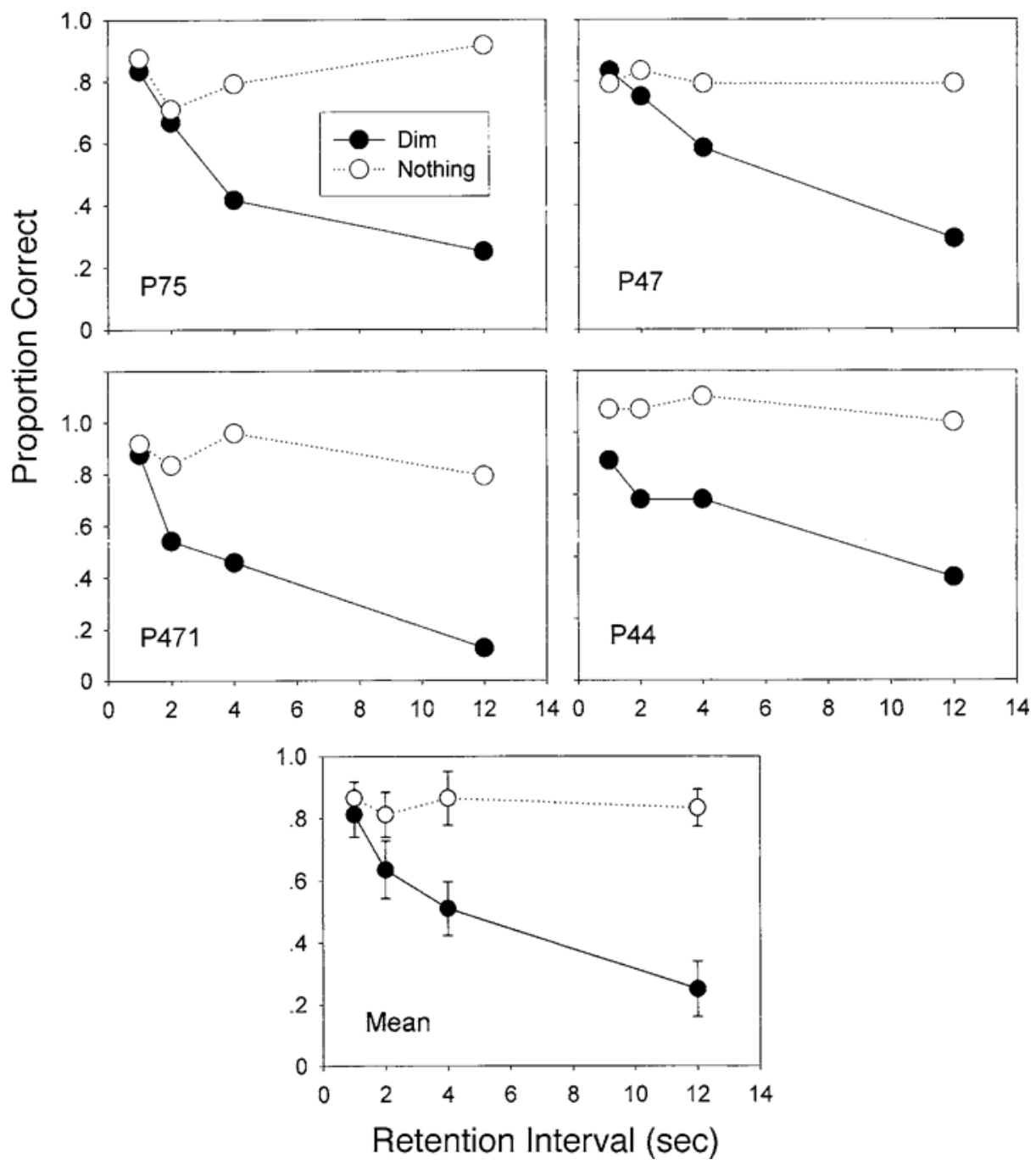

Figure 5. Proportion of correct responses as a function of retention interval on the dim and nosample trials for the 4 birds tested in Experiment 2.

seamlessly into the dark retention interval. Training continued until an average overall accuracy of $85 \%$ correct was maintained across five consecutive sessions, after which probe sessions were introduced. The probe trials were arranged in the same manner as in Experiment 1.

\section{Results and Discussion}

All 4 birds achieved the criterion accuracy level of $85 \%$ correct for five consecutive sessions. Thus, it was clearly possible for the birds to discriminate the dim sample from nothing. Figure 5 presents the results from retention-interval probe trials that were implemented after criterion accuracy was achieved. All 4 birds exhibit a clear asymmetry in the predicted direction. That is, performance on no-sample trials remains flat with increasing retention interval, whereas performance on dim sample trials decreases rapidly to a point well below $50 \%$ correct. An analysis of variance performed on these data revealed a main effect for trial type (dim vs. nothing) $[F(1,3)=55.27, p<.01]$, a main effect for retention interval $[F(3,9)=16.22, p<.01]$, and, most important, an interaction between those two variables $[F(3,9)=27.30$, $p<.01]$. At the longest retention interval, performance following the dim sample was significantly below chance $[t(3)=5.79, p<.01]$. Note that this is the same dim sample that yielded performance that was unaffected by the size of the retention interval (and that was always well above chance) in Experiment 1.

\section{GENERAL DISCUSSION}

The primary contribution of the present research was the demonstration that a predictable asymmetry emerged when the samples in a DMTS task were of unequal salience. Birds trained on a brightness discrimination task in Experiment 1 showed virtually no apparent for- 
getting after presentation of the dim houselight sample but showed rapid forgetting to below $50 \%$ correct after presentation of the bright houselight sample. In Experiment 2, in which the same dim houselight was the more salient sample, performance following the dim sample showed a rapid decline to below $50 \%$ correct.

The simplest explanation for this pattern - an explanation that may apply to a wide range of tasks involving samples of unequal salience-is that pigeons transformed the nominal brightness discrimination task into a detection task in which they responded on the basis of the presence or absence of the more salient sample. If so, one would expect to see no difference in performance on no-sample trials and on trials initiated by the less salient of the two sample stimuli. Indeed, in Experiment 1 here, the birds selected the choice alternative associated with the dim sample about $90 \%$ of the time whether the trial was initiated by a dim sample or by nothing at all on no-sample probe trials. This finding suggests that the dim sample was superfluous in that it did not influence the birds' behavior in any way.

\section{Memory for Asymmetric Samples}

These results are similar to those observed on another task involving samples of (presumably) unequal saliencenamely, samples of short versus long duration. Presumably, a 10-sec (long) houselight is more salient to a bird than a 2 -sec (short) houselight, so the expected result would be an asymmetric forgetting pattern in which performance following the more salient 10 -sec sample declines rapidly to below $50 \%$. Moreover, performance on no-sample probe trials should match performance on trials initiated by the less salient 2 -sec sample. Gaitan and Wixted (2000) found precisely this result.

The same asymmetry observed on the brightness discrimination task has been observed in other versions of the DMTS task that involve samples different in salience (e.g., Colwill, 1984; Fetterman \& MacEwen, 1989; Sherburne \& Zentall, 1993; Spetch \& Wilkie, 1983). These tasks involved delayed discriminations between samples that differ in duration (2-sec vs. 10-sec), sample fixed ratio (FR) requirement (FR 10 vs. FR 40), and food availability (filled vs. empty food hopper). In each case, performance following the more salient sample declined rapidly to below $50 \%$ correct as the retention interval increased while performance following the less salient sample remained accurate. Our claim is that the explanation for the asymmetry observed on the brightness discrimination task applies to these other tasks as well. In all of these experiments, pigeons may have responded based on the presence or absence of the more salient sample. If so, then the task from the pigeons' point of view was not the same as the discrimination task arranged by the experimenter.

Similar considerations may apply in delayed modality discriminations. For example, Wallace, Steinert, Scobie, and Spear (1980) trained rats on a DMTS task in which one sample was visual and the other was auditory. It seems likely that on such a procedure the two samples would not be of equal salience, except by accident. If the two modalityspecific samples did differ in salience, then, for the reasons discussed above, asymmetric forgetting would be expected. Wallace et al. observed a steep forgetting function for visual samples and no apparent forgetting for auditory samples. On the basis of these results, they concluded that visual information decays more rapidly in the rat than does auditory information. That may be true, but another possibility is that the auditory stimuli were not very salient, so the rats responded on the basis of the presence versus absence of the visual sample.

\section{What Is Salience?}

It seems safe to say that intuition easily identifies the less salient sample in many of the tasks that yield asymmetrical forgetting. Thus, for example, the idea that a dim sample is less salient than a bright sample would probably not be especially controversial, which is why we chose that variable for Experiment 1. Still, identifying in advance the less salient sample is not always easy, such as when samples differ in modality. One objective way to test stimulus salience would be to arrange the two samples separately in sample/no-sample tasks. Thus, one task might involve sample trials initiated by a visual stimulus intermixed with no-sample trials. If the animal quickly learns that task to a high level of accuracy, we might say that the visual stimulus is salient because it is so easily discriminated from nothing. A second task might involve sample trials initiated by an auditory stimulus intermixed with no-sample trials. If the animal has trouble learning this task, and if accuracy never reaches high levels, we might say that the auditory stimulus is not very salient (i.e., it is not sufficiently different from nothing to support a high level of performance). When the two samples are pitted against each other in a visual/auditory discrimination task, the asymmetric salience account would predict a steep forgetting function following the more salient visual sample and a flat forgetting function following the less salient auditory sample. In essence, this approach defines stimulus salience as the degree to which the animal can discriminate that stimulus from nothing.

\section{Other Accounts of Asymmetric Forgetting}

The confusion hypothesis. A possible role for stimulus salience in asymmetrical forgetting has not previously been advanced as a critical variable in other theories, but an account proposed by Zentall and his colleagues indirectly suggests the importance of that variable (Sherburne, Zentall, \& Kaiser, 1998). According to their account of the choose-short effect, asymmetrical forgetting functions are observed on duration discrimination tasks because, after a long retention interval, birds become confused as to whether they are in the midst of a retention interval or an ITI. Long ITIs spent in darkness have been a common experience for the birds because they are in effect throughout training, whereas long retention intervals spent in darkness have been an uncommon experience because they occur only during probe sessions. As 
such, the longer the retention interval, the more likely the bird is to believe that an ITI is in effect. When the choice stimuli appear after a long retention interval, the bird may assume that these stimuli appeared during the ITI. Because (from the bird's point of view) no sample was presented, it chooses the choice alternative associated with the sample that is most like nothing (namely, the short sample). It is but a small step to generalize this account to all situations involving samples of unequal salience. In all cases, after a long retention interval, the bird will be inclined to choose the alternative that is most like nothing (because the bird assumes that nothing was presented), which is to say that it will choose the alternative associated with the less salient sample.

The detection account we have proposed here and in other articles is similar to this account, but it differs in one important respect. The detection account does not assume that birds are confused about where they are in a trial. Instead, it assumes that birds attend to and respond on the basis of the more salient sample. That is, they have transformed the task into a presence versus absence task such that the less salient sample is superfluous and can be omitted from the procedure altogether without affecting performance (i.e., performance on nonsalient sample trials and no-sample probe trials should be equivalent). Although our account differs from the confusion hypothesis, it agrees with the confusion hypothesis that one should always see a bias toward the alternative associated with the less salient sample after a long retention interval. The results reported here support this claim and, as such, are consistent with both the detection and confusion accounts.

Subjective shortening. Other theories that have been advanced to explain the choose-short effect require new assumptions to accommodate the asymmetric functions observed on the bright/dim discrimination task. The subjective shortening model, for example, does not apply directly because the samples on this task are of equal duration. However, using reasoning similar to that applied to the duration discrimination task, it could be argued that the bright samples subjectively dimmed with increasing retention interval. That is, the bright sample may have analogically faded along the intensity dimension and, as a result, may have been remembered as being more and more similar to the dim sample stimulus as the retention interval increased.

An even more direct application of the subjective shortening account might hold that bright samples were subjectively longer in duration than dim samples. If so, and if pigeons coded the samples along a subjective temporal dimension as a result, the predicted choose-dim effect would be nothing more than the choose-short effect already explained by the subjective-shortening account. Evidence that bright samples might be perceived as being subjectively longer than dim samples is provided by an elegant study reported by Wilkie (1987) in which pigeons were trained on a 2 -sec versus 10 -sec discrimination pro- cedure. On half the trials, the samples were bright, and on the other half, they were dim. Unreinforced durations of 4,6 , and $8 \mathrm{sec}$ of both dim and bright light were also presented as probes. The birds were less likely to choose the short alternative as the duration of both the bright and dim lights increased (as one would expect), but they were more likely to choose the short alternative with longer durations of the dim light than of the bright light. Wilkie argued that this result implies that the perceived duration of a dim light was shorter than that of a bright light of equal length. Kraemer, Brown, and Randall (1995) reported a similar result for using rats as subjects.

Extrapolating from this, it could be argued that the choose-dim result we observed is simply another demonstration of the widely observed choose-short effect. A disadvantage of this interpretation is that it depends on the assumptions that our birds transformed a brightness discrimination task into a duration discrimination task and the representation of duration shrinks with the passage of time. By instead assuming that birds relied on a detection strategy, a seemingly simpler case can be made. On the other hand, as discussed by Gaitan and Wixted (2000), the subjective shortening model naturally accommodates a wide array of findings over and above the choose-short effect. If one is willing to adopt both assumptions mentioned above, then the subjective shortening model accounts for the choose-dim effect we observed, as well.

The multiple time scale model. Staddon and Higa (1999) proposed an account of duration discrimination performance that assumes that two memory traces are established each time a sample is presented. Thus, for a 2 -sec sample, one trace decay function begins at stimulus onset, and another begins at stimulus offset $2 \mathrm{sec}$ later. The operative psychological variable is the difference in strength between these two forgetting functions when the choice stimuli are presented. That difference is larger for a long sample than for a short one, and the birds are assumed to use the difference variable to solve the task. That is, they learn to choose one alternative when the difference is large and another when it is small. As the retention interval increases, the difference between the two forgetting functions decreases toward zero for both short and long sample trials. Thus, a bias toward the short alternative should emerge.

On the surface, this theory does not appear to predict the asymmetry that is observed in the bright/dim discrimination task used in Experiment 1 because the relevant timing is the same for both samples (i.e., 5 sec elapse from sample onset to sample offset for both). As such, the birds would not be expected to rely on the trace difference variable to solve the task. Although the two trace decay functions associated with the onset and offset of the bright sample may be initially stronger than the two associated with the dim sample, it is not clear that the trace difference would differ for bright and dim samples. Thus, how this theory would be extended to the bright/dim task 
is not clear. The most natural extension of theory would probably closely resemble the subjective dimming explanation mentioned above.

\section{Conclusion}

The findings reported here and the theoretical considerations discussed above represent an attempt to provide a unified account of performance on a wide variety of DMTS tasks involving samples that differ in salience. Whether the proposed detection model can account for the broader array of findings from the relevant literature (e.g., the many other findings that have been taken to support a subjective shortening view of duration discriminations) remains to be seen. At least for the set of studies involving samples of unequal salience followed by a delay interval, the detection model appears to offer a viable and relatively parsimonious explanation.

\section{REFERENCES}

Colwill, R. M. (1984). Disruption of short-term memory for reinforcement by ambient illumination. Quarterly Journal of Experimental Psychology, 36B, 235-258.

Fetterman, J. G., \& MacEwen, D. (1989). Short-term memory for responses: The "choose-small" effect. Journal of the Experimental Analysis of Behavior, 52, 311-324.

GAITAN, S. C., \& WiXTED, J. T. (2000). The role of "nothing" in memory for event duration in pigeons. Animal Learning \& Behavior, 28, 147-161.

Grant, D. S. (1991). Symmetrical and asymmetrical coding of food and no-food samples in delayed matching in pigeons. Journal of Experimental Psychology: Animal Behavior Processes, 17, 186-193.

Kraemer, P. J., Brown, R. W., \& Randall, C. K. (1995). Signal intensity and duration estimation in rats. Behavioural Processes, $\mathbf{3 4}$ 265-268.
Kraemer, P. J., Randall, C. K., \& Brown, R. W. (1997). The influence of stimulus attributes on duration matching-to-sample in pigeons. Animal Learning \& Behavior, 25, 148-157.

McCarthy, D. C., \& Davison, M. (1991). The interaction between stimulus and reinforcer control on remembering. Journal of the Experimental Analysis of Behavior, 56, 51-66.

SCHNEIDER, B. (1987). An interval scale of brightness for the pigeon. Perception \& Psychophysics, 42, 371-376.

Sherburne, L. M., \& Zentall, T. R. (1993). Coding of feature and no-feature events by pigeons performing a delayed conditional discrimination. Animal Learning \& Behavior, 21, 92-100.

Sherburne, L. M., Zentall, T. R., \& Kaiser, D. H. (1998). Timing in pigeons: The choose-short effect may result from pigeons' "confusion" between delay and intertrial intervals. Psychonomic Bulletin \& Review, 5, 516-522.

SPETCH, M. L., \& WILKIE, D. M. (1982). A systematic bias in pigeons' memory for food and light durations. Behaviour Analysis Letters, 2 , 267-274.

Spetch, M. L., \& Wilkie, D. M. (1983). Subjective shortening: A model of pigeons' memory for event duration. Journal of Experimental Psychology: Animal Behavior Processes, 9, 14-30.

STADDON, J. E. R., \& Higa, J. J. (1999). Time and memory: Towards a pacemaker-free theory of interval timing. Journal of the Experimental Analysis of Behavior, 71, 215-251.

Wallace, J., Steinert, P. A., Scobie, S. R., \& Spear, N. E. (1980). Stimulus modality and short-term memory in rats. Animal Learning \& Behavior, 8, 10-16.

WILKIE, D. M. (1987). Stimulus intensity affects pigeons' timing behavior: Implications for an internal clock model. Animal Learning \& Behavior, 15, 35-39.

WIXTED, J. T. (1993). A signal detection analysis of memory for nonoccurrence in pigeons. Journal of Experimental Psychology: Animal Behavior Processes, 19, 400-411.

(Manuscript received June 25, 2003; revision accepted for publication December 1, 2003.) 